\title{
The exercised skeletal muscle: a review
}

\author{
Marina Marini $(1,2)$, Arsenio Veicsteinas $(3,4)$
}

(1) Department of Histology, Embryology, and Applied Biology, University of Bologna, Italy; (2) IIM, Interuniversitary Institute of Myology, Italy; (3) Department of Sport Sciences, Nutrition and Health, University of Milan, Italy; (4) Center of Sport Medicine, Don Gnocchi Foundation, Milan, Italy

\begin{abstract}
The skeletal muscle is the second more plastic tissue of the body - second to the nervous tissue only. In fact, both physical activity and inactivity contribute to modify the skeletal muscle, by continuous signaling through nerve impulses, mechanical stimuli and humoral clues. In turn, the skeletal muscle sends signals to the body, thus contributing to its homeostasis. We'll review here the contribute of physical exercise to the shaping of skeletal muscle, to the adaptation of its mass and function to the different needs imposed by different physical activities and to the attainment of the health benefits associated with active skeletal muscles. Focus will primarily be on the molecular pathways and on gene regulation that result in skeletal muscle adaptation to exercise.
\end{abstract}

Key Words: skeletal muscle, physical exercise, endurance training. force training, metabolic adaptation.

European Journal Translational Myology - Myology Reviews 2010; 20 (3): 105-120

1. Physical exercise and exercise training

2. Fiber type diversity and adaptation to exercise

3. Exercise-induced muscle hypertrophy

4. Metabolic adaptation to exercise

5. Exercise-induced neo-angiogenesis

6. Adaptation of conductive apparatus, sarcoplasmic reticulum and other systems

7. Exercise and generation of reactive oxygen and nitrogen species (RONS)

8. Exercise and skeletal muscle cell damage

9. Major pathways involved in adaptation to exercise and the phenomenon of concurrent training

10. Muscle-to-body signals, hormones, aging

In vertebrates, the skeletal muscle is characterized by several peculiarities that make it one of the more astounding tissues of the body. One of its unusual characteristics resides in its being highly heterogeneous in fiber type, so that it may be looked at as a patchwork of rather different cells; another important feature is its being made up of multinucleated cells, as a result of cell fusion events occurring during development. These factors contribute to make the skeletal muscle the second more plastic tissue of the body - second to the nervous tissue only. In fact, skeletal muscle structure and function can adapt with surprisingly easiness to environmental changes and to different stimuli, ranging from stimuli modifying its contractile activity (inactivity, endurance exercise, denervation, electrical stimulation), stimuli modifying imposed load (resistance exercise, unloading, microgravity) and to other environmental factors such as heat, hypoxia, nutrient availability, growth factors and inflammation mediators. Recent advances allow us to understand the signaling pathways which mediate skeletal muscle hypertrophy and atrophy [98].

The phenomenon of muscle plasticity is observed in all vertebrates, but it displays a great variety in both responsiveness to stimuli and structure-function adaptations. The present overview is limited to humans and rodent species, and will explore the current state of knowledge pertaining to exercise-related adaptations. While it is expected that the same biological processes take place in humans and in rodents, Flück and Hoppeler [27] pointed out in their review that "interspecies congruency of adaptive events is by no means absolute", in particular as the extent and the kinetics of cellular events is concerned. As far as the skeletal muscle plasticity is concerned, it is likely that the most relevant differences between the two models are the age span and the metabolic rate-to-body mass relationship [122]. The purpose of this brief review is to summarize the state of knowledge about exerciseinduced muscle adaptations; since the scientific 


\section{The exercised skeletal muscle}

European Journal Translational Myology - Myology Reviews 20 (3): 105-120, 2010

literature is in rapid expansion, we made the choice of limiting the quoted references mostly to informative reviews that, in turn, will address to the original sources. Readers are also referred to excellent manuals addressing the basic concepts of skeletal muscle structure and mechanisms of contractility and plasticity that have been recently published $[50,63]$.

\section{Physical exercise and exercise training}

Physical exercises affecting the muscle plasticity are usually grouped into two broad categories, those increasing muscle endurance and those increasing muscle strength. Muscle endurance, as the ability of muscle to sustain repeated efforts of relatively low intensity for long periods of time, is also related to the body aerobic capacity, i.e. the efficiency of the cardiovascular and respiratory system in assuming and transporting oxygen. Highly repetitive, low-load exercises favor the adaptation of muscles to a fatigueresistant phenotype.

On the other hand, muscle strength is the ability of muscles to overcome a resistance. This ability is built up by increasing muscle load rather than by increasing the number of repetitions of exercise and is supported by fiber hypertrophy. It mostly relies on the glycolytic metabolism, so that resistance training only slightly improves the aerobic fitness and contributes only marginally to the maintenance of cardiovascular health [4].

When considering the exercise affecting the phenotypic adaptations of muscle, the variables are frequency, intensity, and time (duration), hence the socalled "FIT principle", which popularly describes the fact that their combination will also determine the molecular pathways that are activated in response to exercise and, therefore, determine the final output in terms of endurance and strength or some combination of the two. As Baar [6] points out, the high-intensity training (HIT) studies performed in animals by Terada et al. [112] and in humans by Gibala et al. [31] show that the adaptation to low intensity and long time (duration) training was the same as training at high intensity and short time (duration), i.e. it is dependent on the volume of exercise. Thus, Baar focuses on how variations in load, metabolic stress, and calcium flux are transduced to increases in muscle mass and endurance capacity. Summarizing Baar's hypothesis, if the load is high and the metabolic stress and calcium flux are low, the primary response will be an increase in the rate of protein synthesis and an altered transcriptional profile, resulting in skeletal muscle hypertrophy and increased strength. If the metabolic stress and calcium flux are high, the primary response will be increased mitochondrial mass and oxidative enzymes, resulting in improved fatigue resistance. We will discuss in a later section the molecular pathways that are selectively activated and lead to the specific adaptive responses to endurance or resistance training and their possible interferences (see, for instance, [5, 36]).

\section{Fiber type diversity and adaptation to exercise}

In the mammals, skeletal muscles are constituted of a variety of fiber types, characterized by different structural and functional properties. Their classification, in order of increasing contractile speed and of decreasing reliance on the oxidative metabolism in favor of a glycolytic one, subdivides the skeletal fibers in four major subtypes, which are named after the Myosin Heavy Chain (MHC) isoforms they express. Type I, slow-twitch muscles are specialized for a more continuous activity and a fatigue-resistant phenotype and exploit an oxidative metabolism; they are endowed of a high mitochondrial content and a rich capillary bed, so that they macroscopically appear red. Type IIa fibers are rich in mitochondria and capillaries, so that they too appear red; they stain strongly for succinate dehydrogenase (SDH) but are also rich in glycolytic enzymes, so that they are also classified as fast-twitch oxidative glycolytic fibers and constitute fast fatigue-resistant units. Type IIx (also called IId) fibers are characterized by a more glycolytic metabolism; in rat skeletal muscle, type IIx fibers stain strongly for SDH and are characterized by a velocity of shortening intermediate between Type IIa and IIb, while in humans their staining for SDH is the weakest, since they mostly rely on the glycolytic metabolism; thus, in humans, Type IIx fibers are the fastest and the most fatigable ones. Type IIb fibers constitute the most glycolytic and fastest fibers in rodents (white, fasttwitch glycolytic fibers); in humans, MHC IIb is not expressed.

Immunohistochemistry and other methods showed that muscles are made up of a mosaic (or spectrum) of fiber types with pure or hybrid MHC composition [100]. The pattern of fiber type which characterizes each single muscle is initially established during development, but is maintained through adult life - or shifted toward a different one - by nerve activity and by thyroid hormone. Muscle mechanical activity induces a switch in the expression of myosin heavy chain genes, therefore contributing to the adaptation of the muscle to a given type of physical activity. Chronic phasic high-frequency electrical stimulation, similar to the electric firing of fast motoneurons, can induce a slow-to-fast switch, whereas chronic tonic lowfrequency electrical stimulation, resembling the firing pattern of slow motoneurons, can lead to the fast-toslow switch, although, as pointed out by Schiaffino et al. [100] adaptations occur within the limits imposed by intrinsic differences between muscles and fiber types. Thus, slow muscles have the capacity to adapt in the range $\mathrm{I} \leftrightarrow \mathrm{IIa} \leftrightarrow \mathrm{IIx}$, and fast muscle in the range $\mathrm{IIb} \leftrightarrow \mathrm{IIx} \leftrightarrow \mathrm{IIa}$. This equals to say that, grossly speaking, strength training favors the adaptation of muscles to a slow-to-fast switch, whereas endurance 


\section{The exercised skeletal muscle}

European Journal Translational Myology - Myology Reviews 20 (3): 105-120, 2010

exercises favor the fast-to-slow switch, although such adaptations are usually quantitatively less than those induced by chronic electrical stimulation [83]. As an extreme example of adaptation, world-class marathon runners and ultra-endurance athletes have been found to express as much as $80-90 \%$ of their MHC pool as slow-type MHC Ia, whereas in muscles of elite sprinters and weightlifters IIa/IIx fibers largely predominate [3]. Although it has been suggested that such pattern of MHC gene expression may be mainly ascribed to the genetic predisposition of athletes, it is common opinion that also the specificity of training plays an important role.

Activity-dependent fiber type remodeling is mainly controlled by the calcineurin-NFAT pathway that senses the $\mathrm{Ca}^{2+}$ content of the muscle cell. Calcineurin is a $\mathrm{Ca}^{2+} /$ calmodulin-dependent protein phosphatase that dephosphorylates the transcription factors of the NFAT (nuclear factor of activated $\mathrm{T}$ cells) family, inducing their translocation to the nucleus and subsequent transcriptional activation. Several lines of evidence suggest that calcineurin is the main controller of the activity-dependent expression of the slow MHC gene program in adult muscles (reviewed by [100]), whereas fiber metabolic adaptation and other activitydependent changes are likely to be independent of the calcineurin pathway.

\section{Exercise-induced muscle hypertrophy}

Exercise-induced muscle hypertrophy is observed mostly as a consequence of resistance training. Progressive high-resistance exercise leads to muscle fiber hypertrophy by two partially independent mechanisms, the induction of satellite cell activation and recruitment and the increase of muscle protein synthesis.

A number of signal transduction pathways results into the stimulation of the mTOR (mammalian Target Of Rapamycin) system, which mainly acts by upregulating protein synthesis. The mTOR system is controlled by hormones and growth factors, catecholamines, mechanotransduction signals, phosphatidic acid, amino acids and the energetic state of the muscle [118]. At least two systems oppose such hypertrophy pathway, the myostatin-Smad signalling, which inhibits muscle growth via gene transcription and is in turn controlled by caveolin-3 [79], and the atrophy-related pathways [98].

mTOR is a conserved Ser/Thr kinase of the phosphatidylinositol kinase-related kinase family. It plays a key role in the coordination of important mechanisms such as metabolism and cell growth [121]. In mammals, mTOR exists in two distinct multiprotein complexes, TORC1 and TORC2. TORC1 is a complex comprised of mTOR, regulatory associated protein of mTOR (Raptor), and G protein -subunit-like Gl or mLST8. The TORC2 complex is comprised of mTOR, rapamycin-insensitive companion of mTOR
(Rictor), stress-activated-protein-kinase-interacting protein 1 (SIN1), and G 1. Only the former complex is controlled by exercise and energy-related signals, and is involved in the regulation of cell growth and metabolism, which, in the exercised muscle, result in fiber enlargement [74]. The best understood targets of mTOR are the translation regulators $70-\mathrm{kDa}$ ribosomal protein S6 kinase (p70S6K) S6K1 and 4E-BP1. However, it is important to stress that mTOR controls several additional growth-related processes and inhibits apoptotic signaling. It should be also emphasized that TOR has different functions in different tissues, and pharmacological inhibition of the TORC1 complex may be beneficial in some tissues and detrimental in others [109].

Among the growth promoting factors regulating mTOR, IGF-1 (insulin-like growth factor-1) is the best characterized one. Circulating IGF-1 is produced mainly by the liver under growth hormone control, however stretch and overload induce skeletal muscle to synthesize much of the circulating IGF-1, and also to utilize most of it. As far as increase in mass is concerned, Golspink's group [33] cloned three splice variant of IGF-1 produced in human muscle; one of these, which was called mechano growth factor (MGF) to distinguish it from the other IGFs which have a systemic mode of action, is produced only by active muscle and appears to be the factor that controls local tissue repair, maintenance and remodeling by an autocrine/paracrine mechanism. Notably, dystrophic muscle, unlike normal muscle, is unable to express MGF in response to overload. Moreover, the ability to express MGF has been found to decline markedly during ageing [33]. MGF was also found to directly activate the satellite cell pool and to replenish it after damage [38].

On the other hand, both insulin and the systemic IGF-1 isoforms, produced also by active muscle, are involved in mTOR activation, which in turn leads to the upregulation of protein synthesis in active muscle [115]. Both insulin and IGF-1 activate PI3K, which generates phosphatidylinositol-3,4,5-triphosphate (PIP3). In turn, PIP3 recruits Akt/PKB to the plasma membrane, where it is phosphorylated by at least two distinct kinases, PDK1 and the TORC2 complex. Of the three isoforms present in mammals, exercise is associated with the activation of Aktl $(\mathrm{PKB} \alpha)$, which is upregulated by IGF1 and by still unidentified contraction signals, whereas insulin is known to activate Akt2. Akt upregulates protein synthesis by activating the TORC1 pathway and by inhibiting glycogen synthase kinase $3 \beta$ (GSK3 3 ). The latter enzyme downregulates protein synthesis via eIF2B (eukaryotic initiation factor 2B) inhibition, so that Akt acts by relieving such downregulation. Moreover, Akt inhibits the FoxO pathway, which controls protein degradation [98]. 


\section{The exercised skeletal muscle}

European Journal Translational Myology - Myology Reviews 20 (3): 105-120, 2010

The exercise-induced increase in catecholamines may also play a role in muscle hypertrophy. In fact, activation of beta 2 adrenoreceptors by beta-agonists is known to cause muscle hypertrophy and a slow-to-fast fiber-type switch, by acting through the Akt-mTOR pathway [53].

Mechanical stimuli activate mTOR, without inducing Akt phosphorylation [41]. Muscle activity itself promotes a number of mechanotransduction signals, which are involved in muscle hypertrophic response [124]. In the sarcomere, the basic unit of striated muscles where contractile force originates, dozens of proteins interact with each other and relocalize following mechanical stress or extracellular signals. One of these, the giant protein titin (also called connectin; [115]) is endowed of a kinase domain that regulates the translocation to the nucleus of MURF2, a member of the Muscle-Specific RING-finger proteins. In the nucleus, MURF2 interacts with SRF, the Serum Response Transcription Factor, thus contributing to the regulation of muscle-specific genes [59]. Another source of mechanotransduction signals are the structures connecting the extracellular matrix to the muscle cell membrane (and $\mathrm{z}$ discs) [45]; in particular, integrins have been advocated as important regulators of the mTOR pathway (see discussion in [124]). The expression and/or the phosphorylation state of some components of the dystrophin-associated protein complex (DAPC) were also found to be affected by strength training [55]. It is known that mechanical activity of the muscle increases the concentration of phosphatidic acid (PA), which binds the FRB domain of mTOR and activates p70S6K activity; in turn, PA is generated by the activity of the Phospholipase D (PLD), which hydrolyses phosphatidilcholine in PA and choline and is associated with $\alpha$-actinin and $\beta$ actin. Since $\alpha$-actinin and $\beta$-actin are components of DAPC, it is suggestive to postulate that muscle contraction favors the activation of PLD, and hence PA generation, by favoring its physical dissociation from $\alpha$-actinin and/or $\beta$-actin.

Also nutrients, especially amino acids, activate mTOR. It appears that the amino acid leucine has the most powerful effect on the phosphorylation state of p70S6K in the skeletal muscle. The mechanism by which amino acids activate mTOR is still controversial $[18,32]$, though it is known that the small GTPase Rheb is involved in the activation. In fact, when Rheb is bound to GTP, mTOR is activated. The GTPaseactivating protein that promotes Rheb detachment from mTOR and hence mTOR inactivation is the GAP domain of TSC2. TSC1 and TSC2 (tuberous sclerosis complex 1 and 2), also called hamartin and tuberin respectively, form a complex that represses mTOR. This inhibition on mTOR can be reversed when TSC2 is phosphorylated by $\mathrm{PKB}$, which induces the dissociation of the complex. However, whether high concentrations of amino acids activate $\mathrm{PKB}$ is not well understood [42].

Since mTOR activation results in energy-requiring processes, such as the upregulation of protein synthesis and, in non-muscle cells, in cell cycle promotion, it is not surprising that also the energy status of the cell regulates mTOR activation. Athletes involved in resistance training are encouraged to ingest carbohydrates before a training session, in order to increase muscle mass [114]; moreover, it seems that a minimal insulin concentration - by itself a signal of energy availability - is required for mTOR stimulation by amino acids. On the other hand, a fall in ATP concentration indirectly inhibits the mTOR pathway through the action of AMP kinase (AMPK). AMPK is a sensor of energy shortage; in fact it is activated by high concentration of AMP, and acts by increasing energy availability and decreasing ATP consumption. To this purpose, AMPK, by phosphorylating TSC2, enhances its activity, leading to a decrease in mTOR activity [(46]. Additionally, AMPK can directly phosphorylate the mTOR binding partner Raptor, thus leading to the inhibition of mTOR activity [35]. In this way, the effect of cellular energy stress directly reflects on the mTOR pathway via AMPK activation.

The downstream effectors of mTOR lead to increased ribosome biogenesis and to increased translation. In fact, ribosome biogenesis is an energy consuming process; hence its level is strictly correlated to the energy availability, as well as to the translation needs. TORC1 complex is involved in the activation of (i) RNA polymerase I (Pol I)-dependent rRNA genes, (ii) Pol II-dependent ribosomal protein genes (RP genes), (iii) Pol III-dependent rRNA genes; moreover, it also controls the processing of the $35 \mathrm{~S}$ rRNA precursor [68]. Translation is activated by the TORC1 complex through (i) the activation of p70S6K, which causes the phosphorylation of a subclass of mRNAs encoding elongation factors and ribosomal proteins, thus enhancing the global translation capacity of the cell; (ii) the phosphorylation of 4E-BP1, which releases the eukaryotic initiation factor eIF4E, the limiting component of the multisubunit complex that recruits 40S ribosomal subunits to the 5' end of mRNAs; (iii) the phosphorylation of the eukaryotic elongation factor-2 kinase (EEF2K), which relieves its inhibition on the eukaryotic elongation factor 2 (EEF2). Interestingly, S6K1, one of the p70 kinases, is also involved in the negative regulation of the IGF-1 pathway, thus limiting TOR signaling by a negative feedback loop.

As we have already seen, mTOR function is also inhibited by energy stress (via AMPK-dependent regulation); also RTP801/Redd1 and RTP801L/Redd2, two hypoxia-induced proteins, negatively regulate mTOR activity, by acting on TOR inbitor TSC2, 


\section{The exercised skeletal muscle}

European Journal Translational Myology - Myology Reviews 20 (3): 105-120, 2010

however the details of the underlying mechanisms are still unclear [30].

One relevant pathway that should be considered in the regulation of muscle fiber hypertrophy is that leading to protein degradation that occurs in a number of circumstances such as fasting, disuse and denervation. Evidence obtained by gene profiling studies showed that atrophy is an active process, requiring transcriptional regulation [11, 34]. Briefly, it was found that atrophy signaling acted through the induction of two novel muscle-specific ubiquitinligases, atrogin-1/MAFbx and MuRF1. Although discussing the atrophy pathway goes beyond the scope of this review, we wish to point out the cross-talk connecting the hypertrophy with the atrophy pathways. In fact, it was found that IGF1-Akt signaling is able to block disuse atrophy. When the transcription factors of the FoxO family are phosphorylated by Akt, they are exported from the nucleus to the cytoplasm; it was found, in particular, that FoxO3 induces atrogin1/MAFbx transcription [97]. In addition, FoxO upregulates 4EBP1 and downregulates Raptor and mTOR. Thus, protein breakdown is activated concurrently with protein synthesis inhibition, and viceversa.

Also inflammatory cytokines, such as $\mathrm{TNF} \alpha$, are able to induce protein breakdown through the upregulation of MuRF1, and at the same time they inhibit PI3K-Akt signaling. All the major catabolic pathways are in fact negatively controlled by active mTOR. In fact, mTOR is also involved in the regulation of the autophagy/lysosome pathway and of apoptosis. An excellent discussion of the interconnections between the hypertrophy and the atrophy pathways can be found in a recent review [98].

In the multinucleated muscle fiber, each myonucleus is known to control gene transcription and the subsequent translation over a definite cytoplasm volume [16]. Therefore, all stimuli that determine an increase in the fiber cytoplasmic mass are likely to induce myonuclear addition by satellite cell proliferation and fusion to existing fibers [78]. Bearing in mind that c-fos is expressed at the onset of a mitogenic signaling cascade, it is noteworthy that a single bout of exercise is sufficient to stimulate a more than 20 -fold increase in c-fos mRNA within 4 minutes [14]; this suggests that exercise itself, rather than the need of repairing microtraumas, is the initiating stimulus for satellite cell proliferation. However, mechanisms by which the mechanical stimuli of exercise are transduced into satellite cell activation are still poorly understood. On the other hand, the endocrine context for satellite cell activation and quiescence and the signaling pathways thus controlled are better understood [82, 108, 119]. The stimulating effect of anabolic factors such as nutritional state, androgens, growth hormone, insulin, vitamin $\mathrm{D}$, and pathways requiring nitric oxide release and hepatocyte growth factor-c-met interaction have been acknowledged.

On the other hand, satellite cell activation and proliferation is negatively controlled by the myostatin-Smad signaling pathway. Mutations of the myostatin gene lead to a phenotype of exaggerated muscle hypertrophy, also described in one human child [103]. Myostatin is a member of the TGF superfamily; it binds to activin type I receptors and acts as an inhibitor of muscle growth, by affecting the entry of satellite cells into S-phase. Myostatin gene is upregulated by glucocorticoids and its expression is negatively controlled by exercise, growth hormone and anabolic stimuli. A variety of conditions causing muscle loss are associated with observable increase in myostatin levels, including ageing-associated sarcopenia.

\section{Metabolic adaptation to exercise}

As pointed out above, the energetic characteristics and the enzymatic equipment of muscle fibers are adapted to the specific requirements of a given muscle and to the power output required. Slow skeletal muscles develop lower contractile forces than fast ones, but are able to maintain long-term contractile activity because they rely on oxidative metabolism. On the other hand, fast skeletal muscle fibers develop strong and fast contractions but only for short periods of time because they mainly rely on quickly available but quantitatively limited energy sources, mainly phosphocreatine and glycogen. This makes fast skeletal muscles quickly fatigable, as they replenish their energy reserves mainly through anaerobic glycolysis.

As a consequence of endurance training, in parallel with the fast-to-slow switch in MHC isoforms that has been described above, an increase in skeletal muscle oxidative capacity is observed. Such metabolic adaptation is the result of biochemical cascades that influence gene expression and that are triggered by changes in intramuscular calcium levels, energy availability, plasma concentration of free fatty acids, mechanical stress and local hypoxia. The increase in muscle oxidative capacity involves multiple pathways and is achieved by more sustained fatty acid utilization, mitochondrial biogenesis and higher levels of the oxidative metabolism enzymes, improved glucose transport and neo-angiogenesis.

Enhanced lipid utilization during exercise at moderate intensity in muscles of endurance-trained subjects has been observed, with a selective increase in mRNA levels of enzymes involved in metabolization of intramuscular fatty acids [101] and in their oxidation. In particular, in endurance athletes, it has been reported that skeletal muscle content of MDAC and VLDAC (Medium and Very Long Chain-specific Acyl-CoA Dehydrogenases), involved in beta oxidation of fatty acids, and of peroxisome proliferator-activated receptor-alpha (PPAR $\alpha)$ doubled, despite a limited increase in whole body and plasma fatty acid oxidation [43]. More recently, it has been reported that 


\section{The exercised skeletal muscle}

European Journal Translational Myology - Myology Reviews 20 (3): 105-120, 2010

endurance training in mice [66] and acute exercise in humans [69] increase the expression of PPAR $\beta / \delta$, which is several-fold more abundant than either PPAR $\alpha$ or PPAR $\gamma$ in skeletal muscle and is expressed at higher levels in slow/oxidative compared with fast/glycolytic muscles. The three PPAR isoforms are members of a nuclear receptor superfamily that binds DNA as heterodimers with retinoid $\mathrm{X}$ receptors (RXRs). In mammals they function as metabolic sensors, being activated by lipids and affecting lipid metabolism. In fact, they control a large number of genes encoding proteins involved in mitochondrial metabolism, including those encoding proteins responsible for fatty acid transport and activation, mitochondrial $\beta$-oxidation, and energy uncoupling (reviewed in [29]).

Mitochondria appear to be the key organelle involved in the metabolic adaptation to endurance training. In fact, they host numerous proteins involved in beta oxidation, as well as all proteins involved in oxidative phosphorylation. Mitochondrial DNA content increases in muscles of endurance athletes, together with the level of nuclear transcription factors (NRF-1, NRF-2 and TFAM) that control nuclear-encoded mitochondrial genes. As a result, mitochondrially encoded RNAs as well as nuclear-encoded RNAs are all increased coordinately in the endurance athletes [86].

In turn, nuclear transcription factors that promote mitochondrial biogenesis are activated by the transcriptional co-activator PGC1- $\alpha$. PGC1- $\alpha$ is expressed at lower levels in fast than in slow fibers, but endurance exercise readily increases its expression, allowing it to stimulate mitochondrial biogenesis and synthesis of oxidative enzymes, thus making fasttwitch muscles more resistant to fatigue [64]. The expression of PGC1- $\alpha$ is positively controlled by diverse pathways (reviewed by [39, 40, 100, 123]), such as reactive oxygen species (ROS), calcineurin and $\mathrm{Ca} 2+/$ calmodulin-dependent protein kinases (CaMKs), that sense intracellular $\mathrm{Ca} 2+$ concentration, transcription factors MEF2 (myocyte enhancer factor2 ), which in many tissues control the stress response, p38 mitogen-activated protein kinase (p38MAPK) which in turn requires the activity of the transcription factor ATF2, and AMP kinase. This last activity is triggered by signals of energy shortage [29]. In fact, as already pointed out, AMP kinase controls the pathways that lead to a better utilization of the available energy, including the shift from the glycolytic to the oxidative pathways.

More recently, it was suggested that the ratio $\mathrm{NAD}+/ \mathrm{NADH}$ might be involved in the regulation of skeletal muscle gene expression, after the observation that silent information regulator T1 (SIRT1), an NAD+-dependent deacetylase, deacetylates and activates PGC1- $\alpha$. Since deacetylation by SIRT1 favors PGC1- $\alpha$ translocation to the nucleus, thus promoting its activity even in the absence of transcriptional upregulation, SIRT1 may be regarded as a positive regulator of mitochondrial biogenesis. Cantò et al. [15] have shown that acute exercise, by activating AMPK, modulates NAD + metabolism and SIRT1 mediated-deacetylation of PGC1- $\alpha$. Notably, SIRT1 and PGC1- $\alpha$ mRNA expression have been shown to increase in parallel following caloric restriction and caloric restriction combined with exercise. In different cellular contexts, the NAD+/NADH ratio controls also the activity of AMP kinase and of acetyl-CoA synthase I; these studies collectively suggest that Sirt1 may establish regulatory cross-talk among various energysensing molecules.

Last but not least among the physiological triggers controlling the metabolic adaptation of skeletal muscle to endurance exercise, the central role exerted by local hypoxia that generates during strenuous exercise has been stressed in recent works [2, 19]. By stabilizing the alpha subunit of hypoxia-inducible factor 1 (HIF-1), hypoxia triggers the orchestration of both mitochondrial biogenesis and neo-angiogenesis occurring in the exercised muscle. It should be noted that HIF-1 is directly upstream of both PGC1- $\alpha$ and VEGF induction.

Together with insulin, exercise powerfully stimulates skeletal muscle glucose transport. Both insulin and exercise stimulate the translocation of glucose transporter GLUT4 from an intracellular location to the plasma membrane and t-tubules as well as its neosynthesis. Many lines of evidence suggest that insulin and exercise achieve these results by different pathways (reviewed in [91]). Briefly, the most important mediators of these pathways are AMP kinase, $\mathrm{Ca} 2+/$ calmodulin-dependent protein kinases, protein kinase $\mathrm{C}$, and the AKT substrate of $160 \mathrm{kDa}$ (AS160). This last protein may serve as a point of convergence of both insulin- and contractiondependent signaling in the regulation of glucose uptake [56].

It is important to realize that fiber type transition, mitochondrial biogenesis, increase in GLUT4 and neoangiogenesis can occur independently from each other, thus suggesting distinct signaling mechanisms, although these events do share common triggers and display some overlapping in their activation pathways. The transcriptional modifications leading to the metabolic adaptation of the exercised muscles occur during the first 24 hours of recovery from a training session. At steady state, in the muscles of endurance athletes only one enzyme of the oxidative pathway is upregulated at the mRNA level: GADPH [102]. This suggests that the muscle quickly adjusts itself to variable levels according to the functional demands. Muscle malleability also acts in the detraining phase, when the exercise-induced adaptative modifications are reversed as the training stimulus is no longer present and fiber type spectrum, metabolism, mitochondrial 


\section{The exercised skeletal muscle}

European Journal Translational Myology - Myology Reviews 20 (3): 105-120, 2010

content and vascularization all come to resemble again to the pattern established by basal nerve and hormone activity during adulthood [28].

\section{Exercise-induced neo-angiogenesis}

Exercise training-induced increases in muscle oxidative capacity and energy availability are also accomplished by increasing skeletal muscle blood flow capacity. In fact, the capillary/muscle interface may represent a limiting factor for oxygen delivery and, hence, muscle aerobic capacity. Laughlin and his group (summarized in [60]) showed that at least two primary mechanisms determine the vascular adaptative mechanisms contributing to increases in blood flow capacity: 1) structural remodeling of the vascular tree (capillary bed and arterial tree) and 2) altered vasomotor reactivity of arteries and arterioles; i.e. altered control of conductance. By exploiting the fact that gastrocnemius muscle comprises distinct slow/oxidative and fast/glycolytic regions, they analyzed the effects of different types of exercise training on capillarity in the different regions of the gastrocnemius muscle and showed that exercise training-induced adaptations of capillarity and mitochondrial content are spatially coupled, so that the regions that exhibit increased oxidative capacity also exhibit increased capillarity. That is, endurance training increases capillarity in high oxidative skeletal muscle, but not in low oxidative muscle. In contrast, interval sprint training increases capillarity in fast/glycolytic regions but not in high oxidative muscle tissue. They went on to demonstrate that increases in blood flow capacity are not mediated solely by increased capillarization but also by changes in control of vascular conductance, due to alterations in endothelial and smooth muscle phenotypes and altered control of vascular resistance.

The process of vascular remodeling has been recently reviewed by Egginton [26]. Angiogenesis involves proliferation and migration of endothelial cells to form new capillaries from pre-existing ones. It occurs by different ways in different physiological circumstances, and it is believed to occur as a consequence of exercise as a result of mechanical factors and of the activation of a number of signaling pathways. Also arteriogenesis, involving the remodeling of existing arterial vessels, may play a role in exercise-induced vascular remodeling, and may share many of the growth factors advocated in angiogenesis. Exercise modulates the vasomotor tone, which, in turn, affects vasodilation, by promoting the release of adenosine and, possibly, nitric oxide. Shear stress, transmural pressure and cyclic stretch activate mechanotransduction mechanisms mediated by integrins and associated GTPases. The signal transduction pathways thus stimulated, which recruit different kinases, result in the promotion of angiogenesis.
The major player of angiogenic processes is the Vascular Endothelial Growth Factor, VEGF. VEGF, produced by the endothelial cells, perivascular cells and the host cells, such as skeletal muscle cells, is the ligand of three different receptor tyrosine kinases, localized on the endothelial cells. Ligation of VEGF on its receptors initiates a signaling cascade that includes the activation of $\mathrm{PI}_{3} \mathrm{~K}, \mathrm{PLC} \gamma$ and PKC. In turn, VEGF is positively controlled by different cytokines, growth factors and hypoxia, through the activation of HIF-1 $\alpha$ [65]. It appears that HIF is involved in stretch- but not in shear- induced angiogenesis [25]. The effects of VEGF are mediated by nitric oxide (NO) and prostaglandins that contribute to vascular dilation. NO seems to be involved in shear-, but not in stretchinduced angiogenesis [25]. Other angiogenic or proangiogenic factors, such as FGF, PDGF and TGF- $\beta$, do not appear to be upregulated by exercise. Exercise upregulates VEGF mRNA and protein, as well as the expression of VEGFR1 and 2, in muscle. VEGF is also mobilized and released by active muscle, thus determining an increase in circulating VEGF after acute exercise [57]. However, data evidentiate the existence of a complex relationship between VEGF mRNA and protein levels as well as in VEGF levels after a single bout of exercise or following exercise training (discussed in [10]).

Also proteolytic modification of the extracellular matrix, induced by mechanical deformation during cyclical contractions or muscle overload, are involved in vascular remodeling. In particular, proteolysis of the basement membrane is necessary for capillary sprouting and is carried out by matrix metalloproteinases and other enzymes (plasminogen and the urokinase and tissue plasminogen activators). The process of extracellular matrix degradation must be strictly controlled and coordinated with the angiogenic one [89].

As pointed out above, angiogenesis is mostly induced by endurance training, and is instrumental to the increase in muscle oxidative capacity. In contrast, usually no angiogenesis occurs as a consequence of strength training, where also mitochondrial volume density decreases in proportion to muscle hypertrophy.

\section{Adaptation of conductive apparatus, sarcoplasmic reticulum and other systems}

In a normal muscle fiber, action potentials lead to the depolarization of the sarcolemma, which propagates down the transverse tubules (T-tubules) and activates voltage-gated Cav1.1 $\mathrm{Ca}^{2+}$ channels (also known as Ltype $\mathrm{Ca}^{2+}$ channels or dihydropyridine receptors, DHPRs) on the T-tubules. Voltage-induced conformational changes in DHPRs activate, by some physical interaction, closely apposed $\mathrm{Ca}^{2+}$ release channels known as type 1 ryanodine receptors (RyR1s) on the terminal cisternae of the sarcoplasmic reticulum (SR). $\mathrm{Ca}^{2+}$ release from the SR through RyR1 channels 


\section{The exercised skeletal muscle}

European Journal Translational Myology - Myology Reviews 20 (3): 105-120, 2010

results in a large and rapid rise in cytoplasmic $\left[\mathrm{Ca}^{2+}\right]$ to micromolar levels. By binding to troponin $\mathrm{C}, \mathrm{Ca}^{2+}$ allows myosin and actin to form cross-bridges, leading to the sliding of actin and myosin filaments with respect to each other and the shortening of the sarcomere. This process, known as excitationcontraction (E-C) coupling, results is force generation and muscle contraction [24].

The activity and the status of the different components of the excitation-contraction coupling apparatus are modulated by a number of stable post-transcriptional modifications, including phosphorylation, nitrosylation, and oxidation, that may be downstream of stress-dependent signaling pathways. In effect, repetitive strenuous muscle contractions may be a source of stress for all the cellular components involved in force generation. In particular, repeated cycles of $\left[\mathrm{Ca}^{2+}\right]_{\text {cyt }}$ increases may challenge the survival itself of the fiber; in fact, skeletal muscle contains calpains, $\mathrm{Ca}^{2+}$-activated proteases that may initiate different damage pathways. The mechanism of muscle fatigue, i.e. the mechanism by which repeated strenuous contractions lead to long-lasting decrease in force production, has been recently reviewed $[8 ; 58]$ and seems to be related to E-C uncoupling and acquired $\mathrm{Ca}^{2+}$ leakage from RyR1 channels. The increase in $\left[\mathrm{Ca}^{2+}\right]_{\mathrm{cyt}}$, released from the leaky SR membranes, may locally activate $\mu$-calpains, that may be directly responsible of the E-C uncoupling and also of the structural and morphological damages of the triad system, that have been observed as a consequence of eccentric exercise [111]. Moreover, increases in $\left[\mathrm{Ca}^{2+}\right]_{\text {cyt }}$ may also impose a compensatory activity of SERCA1a ATPase, thus contributing to ATP depletion in the fatiguing muscle.

In a recent study, Duhamel et al. [23] analyzed the establishment of adaptive mechanisms in the muscle $\mathrm{Ca}^{2+}$-cycling properties following three days of intense exercise in humans. Exercise resulted into an increase in SERCA1a but not SERCA2a, meaning that adapting mechanisms protect maximal $\mathrm{Ca}^{2+}$-ATPase activity but not $\mathrm{Ca}^{2+}$ uptake and $\mathrm{Ca}^{2+}$ release. Although the excitation-contraction coupling apparatus comprises dozens of $\mathrm{Ca}^{2+}$-handling proteins, located in the SR lumen, in the SR and in plasma membranes, as well as in the cytosol, to our knowledge other exercise-induced adaptive mechanisms have not been investigated.

Development of strength is an obvious output of exercise training, which particularly concerns sport professionals. Most of the above mentioned mechanisms lead to training-associated development of strength. Additional mechanisms have been recently suggested to contribute to the building up of strength, such as training-induced phosphorylation of myosin regulatory light chains, increased recruitment of higher order motor units, a possible change in pennation angle (i.e. the angle between the longitudinal axes of the whole muscle and its fibers for a pennate-fiber muscle)
[113], changes in neuromuscular control [12] and in endothelial function [21].

7. Exercise and generation of reactive oxygen and nitrogen species (RONS)

During intense activity, the high rate of $\mathrm{O}_{2}$ consumption $\left(\mathrm{VO}_{2}\right)$ in skeletal muscles can cause incomplete oxygen reduction and electron leakage from the electron transfer chain, leading to the generation of superoxide $\left(\mathrm{O}_{2}^{-}\right)$, hydrogen peroxide $\left(\mathrm{H}_{2} \mathrm{O}_{2}\right)$ and hydroxyl radical $(\mathrm{OH})$. Together with nitric oxide and its reaction products, which are generated as well in the active muscle, these reactive nitrogen and oxygen species, collectively known as RONS, may cause the establishment of a condition known as "oxidative stress". According to the definition given by Sies [105], oxidative stress is the "imbalance between oxidants and antioxidants in favor of the oxidants, potentially leading to damage". The first evidence that exercise induced increases in RONS came from electron spin resonance data [20, 48]. Further studies demonstrated that active skeletal muscles released nitric oxide [7], hydroxyl radicals [84], superoxide anions [70] and hydrogen peroxide [71], but only recently it was incontrovertibly demonstrated that mitochondria are the major biological source of ROS production during physical exercise [93]. In fact, there are a number of possible intracellular sources for ROS beside mitochondria, including cytochrome P450, myeloperoxidase, xanthine oxidase, NADH oxidase and peroxisomal oxidative enzymes, all of which may be substantially increased upon exercise.

High levels of ROS generation have been associated to cellular dysfunction and DNA, protein and lipid damage [75]; in muscle cells, they have been suggested to mediate muscle fatigue $[85,88]$. However, exerciseassociated ROS production in the skeletal muscles may be quantitatively limited or, rather, counteracted by anti-oxidants, so that an "unbalance between oxidants and antioxidants in favor of the oxidants", that is oxidative stress, does not necessarily become established. In fact, it was also shown that skeletal muscle adapted to the exercise-induced oxidative environment by upregulating a number of antioxidant enzymes and molecular chaperones [70, 71], that allowed it to be protected against a damaging contraction protocol in vitro [72]. In particular, by using a protocol of muscle stimulation in vivo, a rapid transient reduction in muscle protein thiol content, followed by increases in the activities of superoxide dismutase and catalase and in the content of HSP60, HSP70 and haemoxygenase proteins in both EDL and soleus was found [70-72]. The regulation of the exercise-induced stress response of skeletal muscle has been recently reviewed [76]

On the other hand, a low level of ROS should be regarded as a means of regulating signaling cascades, metabolic and adaptive responses, and the induction of 


\section{The exercised skeletal muscle}

European Journal Translational Myology - Myology Reviews 20 (3): 105-120, 2010

endogenous defense systems, rather than a dangerous challenge leading to cell damage or even cell death (reviewed by Freyssenet [29] and by Powers and Jackson [85]). Increased ROS production is associated with mitochondrial network elongation and branching complexity [54]. Downstream effectors of ROS are a number of key transcription factors, such as NfкB, AP1, HSF, Egr-1, p53. Recently, it has been demonstrated that ROS can induce an increase in PGC1- $\alpha$ transcription via both AMP kinase-dependent and independent pathways [47]. The increase in PGC1- $\alpha$ also causes an increase in NRF-1 and TFAM levels [51]. This directly links ROS production to mitochondrial biogenesis, that is, links the exerciseinduced production of ROS in muscle cells to the cell's shift towards a more oxidative metabolism.

$\mathrm{Nf \kappa B}$ and AP-1 are both thought to be involved in the up-regulation of antioxidant enzymes (discussed in Brooks et al., [13]). In the muscles of chronically exercised male mice, the basal level of $\mathrm{Nf \kappa B}$ binding to DNA was shown to be higher than in the muscles of sedentary ones and this correlated with the ability of exercise-conditioned muscles to detoxify ROS [13], providing protection from ROS-induced damage and from ROS accumulation.

Although the causative mechanisms have not been fully elucidated, it was also shown that muscle's antioxidant capacity, potentiated by chronic endurance exercise, decreases the expression of the pro-apoptotic gene Bax and increases that of the anti-apoptotic Bcl-2 $[1,107]$. A clue for understanding the antiapoptotic effects of exercise may actually come from the observation that exercise upregulates some enzymes involved in DNA repair [87], thus decreasing the likelihood of damaged DNA to initiate a p53-mediated apoptotic cascade. Noteworthy, DNA damage may result from cell exposure to ROS, but DNA damage of long-lived skeletal muscle cells is also associated with aging.

Exercise and its associated ROS generation also transiently induce the production of some inflammatory cytokines in skeletal muscle (reviewed by Pedersen and Febbraio [81] and Scheele et al., [99]). In a similar way as exercise-induced ROS production was initially regarded as a damage-inducing phenomenon, the initial view was that these cytokines were a true sign of exercise-induced inflammation. It now appears that the so-called inflammatory cytokines may have different effects in different humoral contexts; in particular, both upstream and downstream signaling pathways may differ markedly between immune-competent cells, muscle cells and other cells, such as the adipocytes [92]. In healthy, exercised skeletal muscle, these muscle-derived cytokines, renamed "myokines", are now recognized to possess important anti-inflammatory and metabolic properties and to exert multiple beneficial effects, both local and systemic. For instance, IL-6 exerts inhibitory effects on
TNF- $\alpha$ and IL-1 production; IL- 8 acts as an angiogenic factor; IL-15 has been identified as an anabolic factor, which stimulates myogenic differentiation and plays a role in muscle-adipose tissue interaction [81].

All the above described adaptations to ROS may be considered a sort of preconditioning that may explain why trained muscle is less subjected to exerciseinduced damage and underlines the beneficial effects that a moderate amount of exercise-induced ROS may have in the prevention of damage caused by more strenuous or potentially dangerous exercise.

\section{Exercise and skeletal muscle cell damage}

Strenuous and, in particular, eccentric exercise may result in skeletal muscle cell damage, which may manifest itself with LDH release from the cell membrane and/or apoptosis. Both muscle cell damage [95, 96] and exercise-induced oxidative stress [80] have been associated with apoptosis; at the same time, fatigue, which may be per se a symptom of skeletal muscle cell damage, has been associated with oxidative stress [73].

It is nevertheless controversial whether strenuous exercise does in effect cause oxidative stress; in fact, while Selman et al [104] failed to find significant increases in lipid peroxidation products following strenuous exercise in the field mole, Marini et al. [67] reported a significant increase in plasmatic malondialdehyde levels in moderately trained rats. Whether the presence of peroxidation products should be considered a proof of oxidative stress is however questionable. On the other hand, it is possible that the pro-oxidant environment generated in the active muscle, in the presence of inadequate local amounts of anti-oxidants, may in some circumstances lead to skeletal muscle cell damage through a $\mathrm{Ca}^{2+}$-mediated mechanism. This hypothesis is based on the current view that, rather than individual organels, mitochondria may be considered as a complex and dynamic network, which may form junctions with the sarco-endoplasmic reticulum (SR/ER). The close proximity of the two intracellular membrane systems (gap is estimated to be $10-50 \mathrm{~nm}$ wide) allows for collaboration in lipid biosynthesis as well as for local exchanges of signals, including $\mathrm{Ca}^{2+}$ and ROS. In particular, ROS production within mitochondria may directly affect $\mathrm{Ca}^{2+}$-handling molecules on SR/ER. SERCA, RyR1 and IP3R all contain multiple Cys residues, some of which are bona fide sensors of the cell's oxidative stress. Interestingly, low level ROS production by adjacent mitochondria is likely to facilitate SERCA activity, whereas massive ROS generation would affect the functionality of $\mathrm{Ca}^{2+}$ pumps and engage $\mathrm{Ca}^{2+}$ depletion-induced ER stress that may synergize with other effectors to initiate apoptosis [17]. Toxic effects of $\left[\mathrm{Ca}^{2+}\right]_{\text {cyt }}$ increase in skeletal muscle cells have actually been observed in a number of pathological conditions (see, for example, 


\section{The exercised skeletal muscle}

European Journal Translational Myology - Myology Reviews 20 (3): 105-120, 2010

[106]); they are mediated, among others mechanisms, by the activation of calpains and may lead to apoptosis. The fact that oxidative stress may be responsible for muscle soreness and fatigue and even prompt to skeletal muscle damage has suggested that anti-oxidant supplementation may relieve the problem. This intervention has been addressed by a number of studies, which did not reach incontrovertible conclusions. Among the more recent studies, Howatson and van Someren [44] concluded that previous bouts of exercise may have a safer and more efficacious effect on exercise-induced muscle damage than nutritional and pharmacological strategies; Wray et al. [120] examined muscle perfusion during exercise: while anti-oxidant treatment improved skeletal muscle perfusion of the elders, a negative effect in young subjects was found, "adding credence to the concept that some level of free radical concentration is essential for normal function during exercise in young, healthy individuals".

\section{The major pathways involved in adaptation to exercise and the phenomenon of concurrent training}

There is compelling evidence that an active and fit way of life has many important health benefits and that sedentary habits are associated with an increased risk of numerous chronic diseases and decreased longevity (see, for instance, [62]). Current public health recommendations for physical activity combine aerobic exercise with resistance training and flexibility exercises, with the aim of promoting the maintenance of lean body mass, improving muscular strength and endurance, and preserving muscle and junction function, all of which will ultimately promote quality of life (see, for instance, Blair et al., [9]).

However, what is good for the general public may be the worst choice for sport professionals. In fact, the observation that endurance and resistance training cause different adaptations in skeletal muscle suggests that simultaneously training for both strength and endurance results in a compromised adaptation, compared with training for either exercise mode alone. This phenomenon, named "concurrent training", has been first described by Hickson [37]. By utilizing electrical muscle stimulation mimicking endurance or resistance training, Atherton et al. [5] showed that either an AMPK-PGC-1 $\alpha$ - or a PKB-TSC2-mTOR dominated state ensued. In fact, there is molecular evidence for an interference effect when concurrent training is undertaken $[36,77]$. Interestingly, brief repeated sessions of high-intensity interval exercise training (HIT) induce phenotypic changes that resemble traditional endurance training, since signaling through AMP-activated protein kinase and p38 mitogen-activated protein kinase to PGC-1a, leading to mitochondrial biogenesis, tend to prevail over signaling pathways linked to muscle growth, including protein kinase B/Akt and downstream targets p70 ribosomal S6 kinase and 4E binding protein 1 [31].

\section{Muscle-to-body signals, hormones, aging}

From the initial concept that skeletal muscle is the mere object of regulatory clues, finalized to its efficient adaptation to the changing needs of the body, the scientific community is now realizing that the muscle has a central role in regulating its own metabolism and that it is constantly exchanging homeostatic information with the rest of the body. Central to this function is the production of several biologically active substances, including myokines, which participate in cell to-cell and organ-to-organ crosstalk [99].

In the regulation of metabolism, one of the most important organ-to-organ relationships is that between skeletal muscle and adipose tissue. In fact, metabolic changes induced by physical activity contribute to restore a correct caloric equilibrium and to counteract the building up of fat mass. It has been underlined that dynamic changes in adipose tissue and skeletal muscle metabolic and endocrine phenotype contribute to determine the difference between metabolic health and disease [116].

It is also well known that physical exercise is able to improve glucose metabolism and to prevent the onset of insulin resistance [49]; in type 2 diabetes patients, endurance training has proven even more effective than the most widely used anti-diabetic drug, metformin, in controlling glucose metabolism [52].

Interestingly, the widely held opinion that such effects on the control of body fat mass and of glucose metabolism are related to the effect of physical exercise on the energy balance or, rather, to the energy expenditure consequent to physical activity, has been attenuated by a recent paper showing that intake of antioxidants prevented the exercise-mediated increase in insulin sensitivity, thus demonstrating that exercisegenerated ROS have a role in the beneficial effects of physical activity [90].

Another emerging role of resistance training in counteracting the negative effects of type 2 diabete has been underlined by DiPenta et al. [22], who took into consideration the immunomodulatory effects of exercise and concluded that exercise training is able to modulate the pro-inflammatory parameters associated with the disease.

Energy metabolism and physical activity have also an important role in the maintenance of a healthy heart, and, in particular, it has been shown that endurance training decreases the risk for heart failure and is beneficial for heart failure patients. This issue has been recently reviewed [117].

Aging deeply affects the skeletal muscle. Progressive loss of muscle mass (sarcopenia) is one of the most striking phenomena of aging. It is characterized by a deterioration of muscle quantity and quality, leading to a gradual slowing of movement and a decline in 


\section{The exercised skeletal muscle}

European Journal Translational Myology - Myology Reviews 20 (3): 105-120, 2010

strength and power. It is now realized that metabolic adaptations leading to sarcopenia, cachexia and atrophy are only partially overlapping and result from different gene expression and signaling cascades. In sarcopenia, the major changes involve fiber size, mitochondrial homeostatis and apoptosis and are related to a disruption of Akt-mTOR and RhoA-SRF signaling but not Atrogin-1 or MuRF1. A recent review suggests that "resistance training in combination with amino acid-containing nutrition would be the best candidate to attenuate, prevent, or ultimately reverse age-related muscle wasting and weakness" [94].

Within 20 years, more than $20 \%$ of the United States, China, Japan, and Europe population will be older than 65 years and will presumably suffer of age-related diseases and disabilities: metabolic syndrome, cognitive impairment, and physical weakness. As a concluding remark of this review we wish to underline the ability of exercise to offset the age-related decline in both physical and mental fitness (see, for instance, [110] and to significantly contribute to the solution of many important public health problems.

\section{Acknowledgements}

Authors acknowledge the contribution of the Italian Ministry of University and Research (Grant PRIN 2007 protocol number 2007Y4WF3T), Italy and of "Don Gnocchi Foundation", Milan, Italy (Grant "Current Research").

\section{Corresponding Author}

Prof. Marina Marini, Department of Histology, Embryology, and Applied Biology, University of Bologna, Via Belmeloro,8, I-40126 Bologna, Italy Phone: +39 051 2094116; fax +39 051 2094110; Email: marina.marini@unibo.it

\section{References}

[1] Adhihetty PJ, Ljubicic V, Hood DA. Effect of chronic contractile activity on SS and IMF mitochondrial apoptotic susceptibility in skeletal muscle Am. J. Physiol. Endocrinol. Metab. 2007; 292:E748-E755.

[2] Ameln H, Gustafsson T, Sundberg CJ, Okamoto $\mathrm{K}$, Jansson E, Poellinger L, Makino Y. Physiological activation of hypoxia inducible factor-1 in human skeletal muscle. FASEB J. 2005; 19:1009-1011.

[3] Andersen JL, Schjerling P, Saltin B. Muscle, genes and athletic performance. Sci. Am. 2000; 283:48-55.

[4] Armstrong L, Whaley MH, Brubaker PH, Otto RM, ACSM's guidelines for exercise testing and prescription 2006, Lippincott Williams \& Wilkins, USA.
[5] Atherton PJ, Babraj J, Smith K, Singh J, Rennie MJ, Wackerhage H.Selective activation of AMPK-PGC-1alpha or PKB-TSC2-mTOR signaling can explain specific adaptive responses to endurance or resistance traininglike electrical muscle stimulation.FASEB J. 2005; 19: 786-788.

[6] Baar K, The signaling underlying FITness, Appl Physiol Nutr Metab 2009; 34: 411-419.

[7] Balon TW, Nadler JL. Nitric oxide release is present from incubated skeletal muscle preparations. J. Appl. Physiol. 1994; 77:25192521.

[8] Bellinger AM, Mongillo M, Marks AR. Stressed out: the skeletal muscle ryanodine receptor as a target of stress. J. Clin. Invest. 2008; 118:445-453.

[9] Blair SN, LaMonte MJ, Nichaman MZ. The evolution of physical activity recommendations: how much is enough? Am. J. Clin. Nutr. 2004; 79:913S-920S.

[10] Bloor CM. Angiogenesis during exercise and training. Angiogenesis. 2005; 8:263-271.

[11] Bodine SC, Latres E, Baumhueter S, Lai VK, Nunez L, Clarke BA, Poueymirou WT, Panaro FJ, Na E, Dharmarajan K, Pan ZQ, Valenzuela DM, DeChiara TM, Stitt TN, Yancopoulos GD, Glass DJ. Identification of ubiquitin ligases required for skeletal muscle atrophy. Science. 2001; 294:1704-1708.

[12] Bonacci J, Chapman A, Blanch P, Vicenzino B. Neuromuscular adaptations to training, injury and passive interventions: implications for running economy. Sports Med. 2009; 39:903921.

[13] Brooks SV, Vasilaki A, Larkin LM, McArdle A, Jackson MJ. Repeated bouts of aerobic exercise lead to reductions in skeletal muscle free radical generation and nuclear factor kappaB activation. J. Physiol. 2008; 586:39793790.

[14] Cameron-Smith D. Exercise and skeletal muscle gene expression. Clin Exp Pharmacol Physiol 2002; 29:209-213.

[15] Cantó C, Gerhart-Hines Z, Feige JN, Lagouge M, Noriega L, Milne JC, Elliott PJ, Puigserver $\mathrm{P}$, Auwerx J. AMPK regulates energy expenditure by modulating $\mathrm{NAD}(+)$ metabolism and SIRT1 activity. Nature 2009; 458:10561060 .

[16] Cheek DB. The control of cell mass and replication. The DNA unit-a personal 20-year study. Early. Hum. Dev. 1985; 12: 211-239. 


\section{The exercised skeletal muscle}

European Journal Translational Myology - Myology Reviews 20 (3): 105-120, 2010

[17] Csordás G, Hajnóczky G. SR/ER-mitochondrial local communication: calcium and ROS. Biochim. Biophys. Acta 2009; 1787:1352-1362.

[18] Cully M, Downward J. Translational responses to growth factors and stress. Biochem. Soc. Trans. 2009; 37(Pt 1):284-288.

[19] Däpp C, Gassmann M, Hoppeler H, Flück M. Hypoxia-induced gene activity in disused oxidative muscle. Adv. Exp. Med. Biol. 2006; 588:171-188.

[20] Davies KJA, Quintanilla AT, Brooks GA, Parker L. Free radicals and tissue damage produced by exercise. Biochem. Biophys. Res. Commun. 1982; 107:1198-1205.

[21] Di Francescomarino S, Sciartilli A, Di Valerio V, Di Baldassarre A, Gallina S. The effect of physical exercise on endothelial function. Sports Med. 2009; 39:797-812.

[22] DiPenta JM, Green-Johnson JM, Murphy RJ. Type 2 diabetes mellitus, resistance training, and innate immunity: is there a common link? Appl. Physiol. Nutr. Metab. 2007; 32:10251035.

[23] Duhamel TA, Stewart RD, Tupling AR, Ouyang J, Green HJ. Muscle sarcoplasmic reticulum calcium regulation in humans during consecutive days of exercise and recovery. J. Appl. Physiol. 2007; 103:1212-20.

[24] Ebashi, S. Excitation-contraction coupling and the mechanism of muscle contraction. Annu. Rev. Physiol. 1991; 53:1-16.

[25] Egginton S, Zhou AL, Brown MD, Hudlická O. Unorthodox angiogenesis in skeletal muscle. Cardiovasc. Res. 2001; 49:634-646.

[26] Egginton S. Invited review: activity-induced angiogenesis. Pflugers Arch. 2009; 457:963977.

[27] Flück M, Hoppeler H. Molecular basis of skeletal muscle plasticity - from gene to form and function. Rev Physiol Biochem Pharmacol 2003; 146: 159-216.

[28] Flück M. Functional, structural and molecular plasticity of mammalian skeletal muscle in response to exercise stimuli. J. Exp. Biol. 2006;209(Pt 12):2239-2248.

[29] Freyssenet D. Energy sensing and regulation of gene expression in skeletal muscle. J. Appl. Physiol. 2007; 102:529-540.

[30] Frost RA, Huber D, Pruznak A, Lang CH. Regulation of REDD1 by insulin-like growth factor-I in skeletal muscle and myotubes. J. Cell. Biochem. 2009; 108:1192-1202.

[31] Gibala MJ, McGee SL, Garnham AP, Howlett KF, Snow RJ, Hargreaves M. Brief intense interval exercise activates AMPK and p38
MAPK signaling and increases the expression of PGC-1a in human skeletal muscle. J. Appl. Physiol. 2009; 106: 929-934.

[32] Goberdhan DC, Ogmundsdóttir MH, Kazi S, Reynolds B, Visvalingam SM, Wilson C, Boyd $\mathrm{CA}$. Amino acid sensing and mTOR regulation: inside or out? Biochem. Soc. Trans. 2009; 37(Pt 1):248-252.

[33] Goldspink G. Changes in muscle mass and phenotype and the expression of autocrine and systemic growth factors by muscle in response to stretch and overload. J. Anat. 1999; 194: 323 334.

[34] Gomes MD, Lecker SH, Jagoe RT, Navon A, Goldberg AL. Atrogin-1, a muscle-specific Fbox protein highly expressed during muscle atrophy. Proc Natl Acad Sci U S A. 2001; 98:14440-14445.

[35] Gwinn DM, Shackelford DB, Egan DF, Mihaylova MM, Mery A, Vasquez DS, Turk BE, Shaw RJ. AMPK phosphorylation of raptor mediates a metabolic checkpoint. Mol. Cell. 2008; 30: 214-226.

[36] Hawley JA. Molecular responses to strength and endurance training: Are they incompatible?Appl Physiol Nutr Metab 2009; 34: 355-361.

[37] Hickson RC. Interference of strength development by simultaneously training for strength and endurance. Eur. J. Appl. Physiol. Occup. Physiol. 1980; 45: 255-263.

[38] Hill M, Goldspink G. Expression and splicing of the insulin-like growth factor gene in rodent muscle is associated with muscle satellite (stem) cell activation following local tissue damage. J. Physiol. 2003; 549(Pt 2):409-418.

[39] Hood DA, Irrcher I, Ljubicic V, Joseph AM. Coordination of metabolic plasticity in skeletal muscle. J. Exp. Biol. 2006; 209:2265-2275.

[40] Hood DA. Mechanisms of exercise-induced mitochondrial biogenesis in skeletal muscle. Appl Physiol Nutr Metab. 2009;34:465-472.

[41] Hornberger TA, Sukhija KB, Chien S. Regulation of mTOR by mechanically induced signaling events in skeletal muscle. Cell Cycle 2006; 13:1391-1396.

[42] Hornberger TA, Chien S. Mechanical stimuli and nutrients regulate rapamycin-sensitive signaling through distinct mechanisms in skeletal muscle. J Cell Biochem. 2006; 97:1207-1216.

[43] Horowitz JF, Leone TC, Feng W, Kelly DP, Klein S. Effect of endurance training on lipid metabolism in women: a potential role for PPARalpha in the metabolic response to 


\section{The exercised skeletal muscle}

European Journal Translational Myology - Myology Reviews 20 (3): 105-120, 2010

training. Am. J. Physiol. Endocrinol. Metab. 2000;279:E348-E355.

[44] Howatson G, van Someren KA. The prevention and treatment of exercise-induced muscle damage. Med. 2008; 38:483-503.

[45] Ingber DE. Cellular mechanotransduction: putting all the pieces together again. FASEB J.2006; 20: 811-827.

[46] Inoki K, Zhu T, Guan KL. TSC2 mediates cellular energy response to control cell growth and survival. Cell 2003; 115:577-590.

[47] Irrcher I, Ljubicic V, Hood DA. Interactions between ROS and AMP kinase activity in the regulation of PGC-1alpha transcription in skeletal muscle cells. Am. J. Physiol. Cell Physiol. 2009; 296:C116-C123.

[48] Jackson MJ, Edwards HT, Symons MCR. Electron spin resonance studies of intact mammalian skeletal muscle. Biochim. Biophys. Acta 1985; 847:185-190.

[49] James DE, Kraegen EW, Chisholm DJ. Effect of exercise training on whole-body insulin sensitivity and responsiveness. J. Appl. Physiol. 1984; 56:1217-1222.

[50] Jones DA, De Haan A, Round J, Skeletal Muscle -- From Molecules to Movement, 2004, Churchill Livingstone Publ. Philadelphia, PA.

[51] Kang C, O'Moore KM, Dickman JR, Ji LL. Exercise activation of muscle peroxisome proliferator-activated receptor-gamma coactivator-1alpha signaling is redox sensitive. Free Radic Biol Med. 2009; 47:1394-1400.

[52] Kelley DE, Goodpaster BH. Effects of physical activity on insulin action and glucose tolerance in obesity. Med. Sci. Sports Exerc. 1999; 31:S619-S623.

[53] Kline WO, Panaro FJ, Yang H, Bodine SC. Rapamycin inhibits the growth and musclesparing effects of clenbuterol. J. Appl. Physiol. 2007;102:740-747.

[54] Koopman WJ, Verkaart S, Visch HJ, van der Westhuizen FH, Murphy MP., van den Heuvel LW, Smeitink JA, Willems PH. Inhibition of complex I of the electron transport chain causes O2-mediated mitochondrial outgrowth. Am. J. Physiol. 2005; 288, C1440-C1450.

[55] Kosek DJ, Bamman MM. Modulation of the dystrophin-associated protein complex in response to resistance training in young and older men. J. Appl. Physiol. 2008; 104:14761484.

[56] Kramer HF, Taylor EB, Witczak CA, Fujii N, Hirshman MF, Goodyear LJ. Calmodulinbinding domain of AS160 regulates contraction- but not insulin-stimulated glucose uptake in skeletal muscle. Diabetes. 2007; 56:2854-2862.

[57] Kraus RM, Stallings HW 3rd, Yeager RC, Gavin TP. Circulating plasma VEGF response to exercise in sedentary and endurance-trained men. J. Appl. Physiol. 2004; 96:1445-1450.

[58] Lamb GD. Mechanisms of excitationcontraction uncoupling relevant to activityinduced muscle fatigue. Appl. Physiol. Nutr. Metab. 2009; 34:368-372.

[59] Lange S, Ehler E, Gautel M. From A to Z and back? Multicompartment proteins in the sarcomere. Trends Cell Biol. 2006; 16:11-18.

[60] Laughlin MH, Roseguini B. Mechanisms for exercise training-induced increases in skeletal muscle blood flow capacity: differences with interval sprint training versus aerobic endurance training. J. Physiol. Pharmacol. 2008; 59:71-88.

[61] Laughlin $\mathrm{MH}$, Roseguini B. Mechanisms for exercise training-induced increases in skeletal muscle blood flow capacity: differences with interval sprint training versus aerobic endurance training. J. Physiol. Pharmacol. 2008; 59:71-88.

[62] Lee IM, Paffenbarger Jr RS. Associations of light, moderate, and vigorous intensity physical activity with longevity. Am. J. Epidem. 2000; 151: 293-299

[63] Lieber RL, Skeletal Muscle Structure, Function, and Plasticity 2009, Lippincott Williams and Wilkins Publ., Philadelphia, PA.

[64] Lin J, Wu H, Tarr PT, Zhang CY, Wu Z, Boss $\mathrm{O}$, Michael LF, Puigserver P, Isotani E, Olson EN, Lowell BB, Bassel-Duby R, Spiegelman BM. Transcriptional co-activator PGC-1 alpha drives the formation of slow-twitch muscle fibres. Nature 2002; 418:797-801.

[65] Lundby C, Calbet JA, Robach P. The response of human skeletal muscle tissue to hypoxia. Cell Mol Life Sci. 2009; 66:3615-3623.

[66] Luquet S, Lopez-Soriano J, Holst D, Fredenrich A, Melki J, Rassoulzadegan M, Grimaldi PA. Peroxisome proliferator-activated receptor delta controls muscle development and oxidative capability. FASEB J. 2003; 17:2299-2301.

[67] Marini M., Lapalombella R, Margonato V, Ronchi R, Samaja M, Scapin C, Gorza L, Maraldi T, Carinci P,Ventura C, Veicsteinas A. Mild exercise training, cardioprotection and stress gene profile. Eur. J. Appl. Physiol. 2007; 99:503-510.

[68] Martin, D.E., Hall, M.N. The expanding TOR signaling network. Curr. Opin. Cell Biol. 2005; 17:158-166.

[69] Mahoney DJ, Parise G, Melov S, Safdar A, Tarnopolsky MA. Analysis of global mRNA 


\section{The exercised skeletal muscle}

European Journal Translational Myology - Myology Reviews 20 (3): 105-120, 2010

expression in human skeletal muscle during recovery from endurance exercise. FASEB J. 2005; 19:1498-1500.

[70] McArdle A, Pattwell D, Vasilaki A, Griffiths RD, Jackson MJ. Contractile activity-induced oxidative stress: cellular origin and adaptive responses. Am. J. Physiol. Cell Physiol. 2001; 280:C621-C627.

[71] McArdle A, van der Meulen J, Close GL, Pattwell D, Van Remmen H, Huang TT, Richardson AG, Epstein CJ, Faulkner JA, Jackson MJ. Role of mitochondrial superoxide dismutase in contraction-induced generation of reactive oxygen species in skeletal muscle extracellular space. Am. J. Physiol. Cell Physiol. 2004; 286:C1152-C1158.

[72] McArdle F, Spiers S, Aldemir H, Vasilaki A, Beaver A, Iwanejko L, McArdle A, Jackson MJ. Preconditioning of skeletal muscle against contraction-induced damage: the role of adaptations to oxidants in mice. J. Physiol. 2004; 56:233-244.

[73] McClung JM, Deruisseau KC, Whidden MA, Van Remmen H, Richardson A, Song W, Vrabas IS, Powers SK. Overexpression of antioxidant enzymes in diaphragm muscle does not alter contraction-induced fatigue or recovery. Exp. Physiol. 2010; 95:222-231.

[74] Miyazaki M, Esser KA. Cellular mechanisms regulating protein synthesis and skeletal muscle hypertrophy in animals. J Appl Physiol. 2009;106:1367-1373.

[75] Moylan, J.S. and Reid, M.B. Oxidative stress, chronic disease, and muscle wasting. Muscle Nerve 2007; 35:411-429.

[76] Morton JP, Kayani AC, McArdle A, Drust B. The exercise-induced stress response of skeletal muscle, with specific emphasis on humans. Sports Med. 2009; 39:643-662.

[77] Nader GA. Concurrent strength and endurance training: from molecules to man. Med. Sci. Sports Exerc. 2006; 38:1965-1970.

[78] O’Connor RS, Pavlath GK, McCarthy JJ, Esser KA. Point:Counterpoint: Satellite cell addition is/is not obligatory for skeletal muscle hypertrophy. J. Appl. Physiol: 2007; 103: 1107.

[79] Ohsawa Y, Okada T, Kuga A, Hayashi S, Murakami T, Tsuchida K, Noji S, Sunada Y. Caveolin-3 regulates myostatin signaling. Minireview. Acta Myol. 2008;27:19-24.

[80] Phaneuf S, Leeuwenburgh C. Apoptosis and exercise. Med. Sci. Sports Exerc. 2001; 33:393396.
[81] Pedersen BK, Febbraio MA. Muscle as an endocrine organ: focus on muscle-derived interleukin-6. Physiol Rev. 2008; 88:1379-1406.

[82] Petrella JK, Kim JS, Mayhew DL, Cross JM, Bamman MM. Potent myofiber hypertrophy during resistance training in humans is associated with satellite cell-mediated myonuclear addition: a cluster analysis. J. Appl. Physiol. 2008; 104:1736-1742.

[83] Pette D, Staron RS, Transitions of muscle fiber phenotypic profiles. Histochem. Cell. Biol. 2001;115:359-372.

[84] Powers SK, Ji LL, Leeuwenburgh C. Exercise training-induced alterations in skeletal muscle antioxidant capacity: a brief review. Med. Sci. Sports Exerc. 1999; 31:987-997.

[85] Powers SK, Jackson MJ. Exercise-induced oxidative stress: cellular mechanisms and impact on muscle force production. Physiol. Rev. 2008; 88:1243-1276.

[86] Puntschart A, Claassen H, Jostarndt K, Hoppeler H, Billeter R. mRNAs of enzymes involved in energy metabolism and mtDNA are increased in endurance-trained athletes. Am. J. Physiol. 1995; 269:C619-C625.

[87] Radák Z, Naito H, Kaneko T, Nakamoto H, Takahashi R, Cardozo-Pelaez F., Goto S. Exercise training decreases DNA damage and increases DNA repair and resistance against oxidative stress of proteins in aged skeletal muscle. Pflugers Arch. 2002; 445:273-278.

[88] Reid, M.B. Free radicals and muscle fatigue: of ROS, canaries, and the IOC. Free Radic. Biol. Med. 2008; 44:169-179.

[89] Rivilis I, Milkiewicz M, Boyd P, Goldstein J, Brown MD, Egginton S, Hansen FM, Hudlická O, Haas TL. Differential involvement of MMP2 and VEGF during muscle stretch- versus shear stress-induced angiogenesis. Am. J. Physiol. Heart Circ. Physiol. 2002; 283:H1430-H1438.

[90] Ristow M, Zarse K, Oberbach A, Klöting N, Birringer M, Kiehntopf M, Stumvoll M, Kahn $\mathrm{CR}$, Blüher M. Antioxidants prevent healthpromoting effects of physical exercise in humans. Proc. Natl. Acad. Sci. U S A. 2009; 106:8665-8670.

[91] Röckl KSC, Witczak CA, Goodyear LJ. Signaling mechanisms in skeletal muscle: acute responses and chronic adaptatiuons to exercise. Life 2008; 60:145-153.

[92] Rosa Neto JC, Lira FS, Oyama LM, Zanchi NE, Yamashita AS, Batista ML Jr, Oller do Nascimento CM, Seelaender M. Exhaustive exercise causes an anti-inflammatory effect in skeletal muscle and a pro-inflammatory effect 


\section{The exercised skeletal muscle}

European Journal Translational Myology - Myology Reviews 20 (3): 105-120, 2010

in adipose tissue in rats. Eur. J. Appl. Physiol. 2009; 106:697-704.

[93] Sahlin K, Shabalina IG, Mattsson CM, Bakkman L, Fernström M, Rozhdestvenskaya Z, Enqvist JK, Nedergaard J, Ekblom BT, Tonkonogi M. Ultra-endurance exercise increases the production of reactive oxygen pecies in isolated mitochondria from human skeletal muscle. J .Appl. Physiol. 2010 Jan 28. [Epub ahead of print]

[94] Sakuma K, Yamaguchi A.Molecular Mechanisms in Aging and Current Strategies to Counteract Sarcopenia.Curr. Aging Sci. 2010 Feb 17. [Epub ahead of print]

[95] Salmons S. Muscle Damage. Basic Appl Myol 1994; 4: 1-438

[96] Sandri M, Carraro U. Apoptosis of skeletal muscles during development and disease. Int. J. Biochem. Cell Biol. 1999;31:1373-1390.

[97] Sandri M, Sandri C, Gilbert A, Skurk C, Calabria E, Picard A, Walsh K, Schiaffino S, Lecker SH, Goldberg AL. Foxo transcription factors induce the atrophy-related ubiquitin ligase atrogin-1 and cause skeletal muscle atrophy. Cell. 2004;117:399-412.

[98] Sandri M., Signaling in Muscle Atrophy and Hypertrophy, Physiology 2008; 23: 160-170.

[99] Scheele C, Nielsen S, Pedersen BK. ROS and myokines promote muscle adaptation to exercise. Trends Endocrinol. Metab. 2009; 20:95-99.

[100] Schiaffino S, Sandri M, Murgia M. Activitydependent signaling pathways controlling muscle diversity and plasticity. Physiology 2007; 22:269-278.

[101] Schmitt B, Flück M, Décombaz J, Kreis R, Boesch C, Wittwer M, Graber F, Vogt M, Howald H, Hoppeler H. Transcriptional adaptations of lipid metabolism in tibialis anterior muscle of endurance-trained athletes. Physiol. Genomics 2003; 15:148-157.

[102] Schmutz S, Däpp C, Wittwer M, Vogt M, Hoppeler H, Flück M. Endurance training modulates the muscular transcriptome response to acute exercise. Pflugers Arch. 2006; 451:678-687.

[103] Schuelke M, Wagner KR, Stolz LE, Hubner C, Riebel T, Komen W, Braun T, Tobin JF, Lee SJ. Myostatin mutation associated with gross muscle hypertrophy in a child. N. Engl. J. Med. 2004; 350: 2682-2688.

[104] Selman C, McLaren JS, Collins AR, Duthie GG, Speakman JR. Antioxidant enzyme activities, lipid peroxidation, and DNA oxidative damage: the effects of short-term voluntary wheel running Arch. Biochem. Biophys. 2002; 401:255-261.

[105] Sies H (1991) Oxidative stress. II. Oxidants and antioxidants. Academic Press, London

[106] Sirvent P, Mercier J, Lacampagne A.New insights into mechanisms of statin-associated myotoxicity. Curr. Opin. Pharmacol. 2008; 8:333-338.

[107] Siu PM, Bryner RW, Martyn JK, Alway SE. Apoptotic adaptations from exercise training in skeletal and cardiac muscles. FASEB J. 2004; 18:1150-1152.

[108] Solomon AM, Bouloux PM. Modifying muscle mass - the endocrine perspective. J. Endocrinol. 2006; 191:349-360.

[109] Stanfel MN, Shamieh LS, Kaeberlein M, Kennedy BK. The TOR pathway comes of age.Biochim Biophys Acta. 2009;1790:10671074.

[110] Strijk JE, Proper KI, van der Beek AJ, van Mechelen W. The Vital@Work Study. The systematic development of a lifestyle intervention to improve older workers' vitality and the design of a randomised controlled trial evaluating this intervention. BMC Public Health. 2009;10:408.

[111] Takekura H, Fujinami N, Nishizawa T, Ogasawara H, Kasuga N. Eccentric exerciseinduced morphological changes in the membrane systems involved in excitationcontraction coupling in rat skeletal muscle. J. Physiol. 2001; 533:571-583.

[112] Terada S, Yokozeki T, Kawanaka K, Ogawa K, Higuchi M, Ezaki O, Tabata I. Effects of highintensity swimming training on GLUT-4 and glucose transport activity in rat skeletal muscle. J. Appl. Physiol. 2001; 90: 2019-2024.

[113] Tillin NA, Bishop D. Factors modulating postactivation potentiation and its effect on performance of subsequent explosive activities. Sports Med. 2009; 39:147-166.

[114] Tipton KD, Rasmussen BB, Miller SL, Wolf SE, Owens-Stovall SK, Petrini BE, Wolfe RR Timing of amino acid-carbohydrate ingestion alters anabolic response of muscle to resistance exercise. Am. J. Physiol 2001; 281:E197-206.

[115] Tskhovrebova, L. and Trinick, J. Titin: properties and family relationships. Nat. Rev. Mol. Cell Biol. 2003; 4: 679-689.

[116] Ukropec J, Ukropcova B, Kurdiova T, Gasperikova D, Klimes I. Adipose tissue and skeletal muscle plasticity modulates metabolic health. Arch. Physiol. Biochem.. 2008; 114:357-368. 


\section{The exercised skeletal muscle}

European Journal Translational Myology - Myology Reviews 20 (3): 105-120, 2010

[117] Ventura-Clapier R. Exercise training, energy metabolism, and heart failure. Appl. Physiol. Nutr. Metab. 2009; 34:336-339.

[118] Wackerhage H, Ratkevicius A. Signal transduction pathways that regulate muscle growth, Essays Biochem 2008; 44: 99-108.

[119] Wozniak AC, Kong J, Bock E, Pilipowicz O, Anderson JE. Signaling satellite-cell activation in skeletal muscle: markers, models, stretch, and potential alternate pathways. Muscle Nerve 2005; 31:283-300.

[120] Wray DW, Nishiyama SK, Monnet A, Wary C, Duteil SS, Carlier PG, Richardson RS. Antioxidants and aging: NMR-based evidence of improved skeletal muscle perfusion and energetics. Am. J. Physiol. Heart Circ. Physiol. 2009; 297:H1870-H1875.

[121] Wullschleger S, Loewith R, Hall MN. TOR signaling in growth and metabolism. Cell 2006; 124:471-484.

[122] Weibel ER, Physiology: the pitfalls of power laws, Nature 2002; 417: 131-132.

[123] Yan Z. Exercise, PGC-1alpha, and metabolic adaptation in skeletal muscle. Appl. Physiol. Nutr. Metab. 2009; 34:424-427.

[124] Zanchi NE, Lancha AH Jr. Mechanical stimuli of skeletal muscle: implications on $\mathrm{mTOR} / \mathrm{p} 70 \mathrm{~s} 6 \mathrm{k}$ and protein synthesis. Eur J Appl Physiol. 2008; 102:253-263. 